\title{
3D Moment Invariant Based Morphometry
}

\author{
J.-F. Mangin ${ }^{1,2}$, F. Poupon ${ }^{1,2}$, D. Rivière ${ }^{1,2}$, A. Cachia ${ }^{1,2}$, D.L. Collins ${ }^{3}$, \\ A.C. Evans ${ }^{3}$, and J. Régis ${ }^{4}$ \\ 1 Service Hospitalier Frédéric Joliot, CEA, 91401 Orsay, France \\ mangin@shfj.cea.fr, http://anatomist.info \\ 2 Institut Fédératif de Recherche 49 (Imagerie Neurofonctionnelle), Paris \\ 3 Montreal Neurological Institute, McGill University, Montreal \\ ${ }^{4}$ Service de Neurochirurgie Fonctionnelle et Stereotaxique, CHU La Timone, Marseille
}

\begin{abstract}
This paper advocates the use of shape descriptors based on moments of 3D coordinates for morphometry of the cortical sulci. These descriptors, which have been introduced more than a decade ago, are invariant relatively to rotations, symmetry and scale and can be computed for any topology. A rapid insight of the derivation of these invariants is proposed first. Then, their potential to characterize shapes is shown from a principal component analysis of the 12 first invariants computed for 12 different deep brain structures manually drawn from 7 different brains. Finally, these invariants are used to find some correlates of handedness among the shapes of 116 different cortical sulci automatically identified in 144 brains of the ICBM database.
\end{abstract}

\section{Introduction}

This paper advocates the use of shape descriptors based on moments of 3D coordinates for morphometry purpose. These 3D descriptors, which are invariant relatively to rotation, symmetry and scale, have been introduced more than a decade ago [8], as a 3D extension to the $2 \mathrm{D}$ moment invariants widely used in pattern recognition [6]. These 3D moment invariants have not gained a lot of attention in the medical imaging community. In our opinion, however, they provide a powerful way to perform global morphometry of anatomical entities because they impose no constraints on the object's topology. Therefore, they appear as an interesting alternative to the spherical harmonics based approach, which implies simply connected objects for 2D parameterization of the surface [15]. While the theoretical derivation of the invariants from the coordinate moments is complex, they can be computed in a simple and robust way from a binary volume based description of the objects of interest. This simplicity of use makes these invariants good candidates for mining large databases of objects before using more sophisticated shape analysis tools providing locality to the study [11.5].

In this paper, the interest of the 3D moment invariants is illustrated through the study of the shapes of the cortical sulci of 144 subjects of the ICBM database. A previous study has shown some correlates of handedness on the global size of some of the sulci of the motor and premotor areas [10]. These correlates are supposed to stem from handedness-related discrepancies in the pressure to increase the local folding induced by discrepancies in the development of the surrounding cortical areas. This first study has shown that sulcus based morphometry is a compelling complement to the usual voxel 
based morphometry. VBM, indeed, could not reveal any significant handedness-related result [16], although the existence of some correlates could be forecast from previous manual studies. Such disagreement between both morphometry strategies may stem from the loss of statistical power induced by the non perfect gyral matching performed by the spatial normalization underlying VBM. Therefore, sulcus based morphometry may become a new probe to test the assumption that certain neuroanatomical structures may be preferentially modified by particular cognitive skills or diseases.

The first study mentioned above was relying on the size of the pieces of skeleton used to represent the sulci of interest [9]. This measure of size is analogous to the volume calculation used in standard morphometric studies. It is evident that sulcus characterization by size does only capture one of the multiple aspects of the folding patterns [5]. We propose the use of 3D moment invariants as a richer description of the sulcus shapes. They appear especially adapted to sulcus morphometry, because the numerous and variable sulcus interruptions prevent a simple parameterization strategy for most of the sulci [1,7]. The next section provides an insight into the origin of the invariants, and a few experiments about their invariance properties and their slow variations in the shape space. The last section reports some invariant-based results relative to the handednesscorrelate study of about 116 sulci automatically labeled in each of 144 brains by a system described elsewhere [14].

\section{3D Moment Invariants}

The 3D moments of order $n=p+q+r, n \in \mathbb{N}$ of a 3D density function $\rho(x, y, z)$ are defined by

$$
m_{p q r}=\int_{-\infty}^{+\infty} \int_{-\infty}^{+\infty} \int_{-\infty}^{+\infty} x^{p} y^{q} z^{r} \rho(x, y, z) d x d y d z
$$

In the following, $\rho(x, y, z)=1$, because we deal with objects defined by binary images. The moments of order higher than 3 will not be considered in this paper for the sake of simplicity, but the derivation of moment invariants is theoretically possible for any order. By discarding moments of order higher than 3, we get a small set of global descriptors which should embed simple shape information like bending, tapering, pinching, etc... The derivation of the invariants aims at filtering out the influence of localization, orientation and scale on the 3D moments in order to obtain "pure shape" descriptors. Translation invariance is simply obtained using central moments. In the following, for the sake of clarity, the origin of the coordinate system is assumed to be at the centroid of the object and the corresponding central moments will be written $M_{p q r}$. As shown in [6] for the 2D case, the similitude invariance is obtained by normalizing moments with the suitable power of the volume $M_{000}$. Therefore, in the following we consider

$$
\mu_{p q r}=\frac{M_{p q r}}{M_{000} \frac{p+q+r}{3}+1} .
$$

Rotation invariants can be derived from group theory techniques usual in quantum mechanics [4]. When a rotation is applied to the underlying object, central moments of 
order $n$ are transformed into linear combinations of moments of the same order. This result stems from the fact that homogeneous polynomials of order $n$ form a subspace $\mathcal{P}_{n}$ of the functions of $\mathbb{R}^{3}$ stable under the rotation group. The coefficients of the linear combinations mentioned above are the matrix elements of a representation of the 3D rotation group (corresponding to a group homomorphism) [4]. This representation is reducible, which means that $\mathcal{P}_{n}$ can be decomposed into a direct sum of smaller subspaces stable under the rotation group. Rotation invariants stem from the finest possible decomposition leading to irreducible representations. In this new basis, the effect of the rotation operator on a vector of $\mathcal{P}_{n}$ corresponds to a block diagonal matrix

$$
D=\left(\begin{array}{cccc}
D_{0} & & & \\
& \left(D_{1}\right) & & \\
& & \ddots & \\
& & & \left(D_{L}\right)
\end{array}\right),
$$

where $D_{0}, \ldots, D_{L}$ are irreducible representations [4].

The basis corresponding to this decomposition is the basis of harmonic polynomials $y_{l}^{m}=r^{l} Y_{l}^{m}, l=0, \ldots, L, m=-l, \ldots, l$ where $Y_{l}^{m}$ are the spherical harmonics and $r=\sqrt{x^{2}+y^{2}+z^{2}}$. In the following, $\mathcal{E}_{l}$ denotes the subspace defined by harmonic polynomials of order $l$. The space $\mathcal{P}_{n}$ of homogeneous polynomials of order $n$ decomposes itself into subspaces $\mathcal{E}_{n}, \mathcal{E}_{n-2}, \mathcal{E}_{n-4}$, etc. . For instance $\mathcal{P}_{2}$ splits into $\mathcal{E}_{2}$ and $\mathcal{E}_{0}$, $\mathcal{P}_{3}$ splits into $\mathcal{E}_{3}$ and $\mathcal{E}_{1}$, etc... In the new basis of $\mathcal{P}_{n}$, moments $\mu_{p q r}$ are transformed into complex moments $\nu_{l}^{m}$ and rotation invariants are derived from $\nu_{l}^{m}$ using tensor products, which may be understood as a generalization of scalar or vector products [8, 4].

Rotation invariants are inferred from all the possible applications of the tensor product to $\nu_{0}^{m}, \nu_{1}^{m}, \nu_{2}^{m}$ and $\nu_{3}^{m}$ yielding rank-0 tensors, namely scalars which are the 3D moment invariants. These invariants turn out to be homogeneous polynomials of central moments. Because of various symmetries, the tensor products results only in twelve invariants $I_{\beta}^{\alpha}$, where $\alpha$ denotes the order of the underlying central moments and $\beta$ denotes the subspace indices of the different tensors used in the application of the products [8, 12]. Thus, we get four norms $I_{00}^{2}, I_{11}^{3}, I_{22}^{2}$, and $I_{33}^{3}$, five scalar products and norms of new tensors $I_{222}^{2}, I_{3111}^{3}, I_{3131}^{3}, I_{3331}^{3}$, and $I_{3333}^{3}$, and three last invariants derived by combining the second- and third-order moments $I_{112}^{2,3}, I_{312}^{2,3}$, and $I_{332}^{2,3}$. Since moment invariants are expressed by homogeneous polynomials, they can be finally reduced by the suitable power of the invariant [2]. Then each invariant $I_{\beta}^{\alpha}$ is transformed in

$$
\tilde{I}_{\beta}^{\alpha}=\operatorname{sign}\left(I_{\beta}^{\alpha}\right) \cdot\left|I_{\beta}^{\alpha}\right|^{1 / d},
$$

where $\tilde{I}_{\beta}^{\alpha}$ is the reduced moment invariant and $d$ the polynomial degree.

In order to check that the theoretical properties of invariance stand for discrete representations of objects relying on binary images, two simple shapes have been resampled with 28 different orientations. Each invariant has been computed for each orientation. The obtained standard deviations are almost negligible relatively to the means, which shows that the rotation invariance is respected (see Fig 11). The standard deviations are higher for the ventricle than for the pinched superquadric, which can be understood from the fact that the resampling induces more modifications of the thinest shape. 


\begin{tabular}{|c|c|c|c|c|}
\hline Invariants & \multicolumn{2}{|c|}{ Superquadric } & \multicolumn{2}{|c|}{ Ventricle } \\
\cline { 2 - 5 } & $m$ & $\sigma$ & $m$ & $\sigma$ \\
\hline$\tilde{I}_{00}^{2}$ & 0.569 & 0.0002 & 1.572 & 0.002 \\
$\tilde{I}_{22}^{2}$ & 0.271 & 0.0004 & 1.147 & 0.001 \\
$\tilde{I}_{222}^{2}$ & -0.299 & 0.0005 & -1.263 & 0.002 \\
$\tilde{I}_{33}^{3}$ & -0.233 & 0.0004 & -0.732 & 0.001 \\
$\tilde{I}_{11}^{3}$ & -0.235 & 0.0008 & -0.825 & 0.003 \\
$\tilde{I}_{3333}^{3}$ & 0.223 & 0.0004 & 0.711 & 0.001 \\
$\tilde{I}_{3131}^{3}$ & 0.177 & 0.0004 & 0.581 & 0.002 \\
$\tilde{I}_{3331}^{3}$ & -0.198 & 0.0004 & -0.621 & 0.002 \\
$\tilde{I}_{3111}^{3}$ & -0.176 & 0.0005 & -0.555 & 0.005 \\
$\tilde{I}_{332}^{2,3}$ & 0.231 & 0.0004 & 0.771 & 0.001 \\
$\tilde{I}_{312}^{2,3}$ & -0.194 & 0.0004 & -0.677 & 0.001 \\
$\tilde{I}_{112}^{2,3}$ & 0.189 & 0.0007 & 0.540 & 0.019 \\
\hline
\end{tabular}

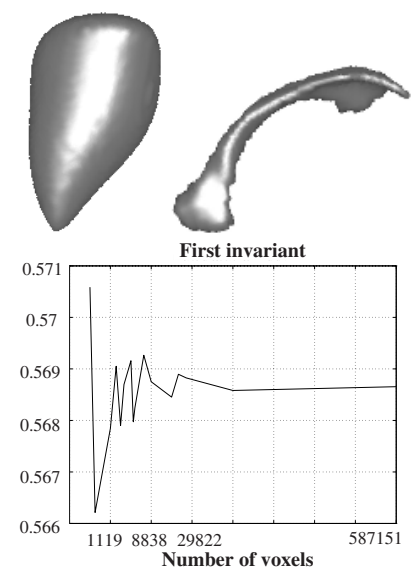

Fig. 1. left: Means and standard deviations of the twelve invariants for 28 different orientations of the two objects visualized in the figure. right: Typical variations observed for different sampling of the pinched superquadric. NB: in all figures, for the sake of visualization, voxel-based objects are triangulated before $3 D$ rendering.

In order to check that the 3D moment invariants vary sufficiently slowly in the shape space to be interesting as shape descriptors, we have performed a simple principal component analysis of the invariants obtained for 6 different kinds of shapes corresponding to deep brain nuclei and lateral ventricles. These objects have been manually drawn by a neuroanatomist in the two hemispheres of 7 different brains and can be visualized in Fig. 2] Plotting the 84 objects in a chart corresponding to the three first axes yielded by the PCA shows that the instances of each anatomical entity gather in one localized region of the shape space, as described by the invariants. Furthermore, the regions corresponding to two nuclei with similar shapes, are closed in this space. This is for instance clear for the pairs (caudate nucleus, lateral ventricle), or (putamen, globus pallidus). These properties have been used previously to design shape probability distributions embedded in a Bayesian framework to bias a multi-object deformable model dedicated to brain basal ganglia [13].

\section{Result}

The large scale morphometric studies of the handedness correlates has been performed on a sulcus-by-sulcus basis, each sulcus being identified automatically by a computer vision system freely available on http://anatomist.info. The subjects scanned were 144 unselected normal volunteers previously used for one of the VBM studies mentioned above [16]. On a short handedness questionnaire, 14 subjects were dominant for lefthand use on a number of tasks; the remaining 130 subjects preferred to use their right hand. The $144 \mathrm{~T} 1$-weighted brain volumes were stereotaxically transformed using nine parameters [3] to match the Montreal Neurological Institute 305 average template. The cortical folds were then automatically extracted using a 3D skeletonization [9]. Finally, 58 cortical sulci were recognized in each hemisphere [14]. 
Axis 2 versus axis 1

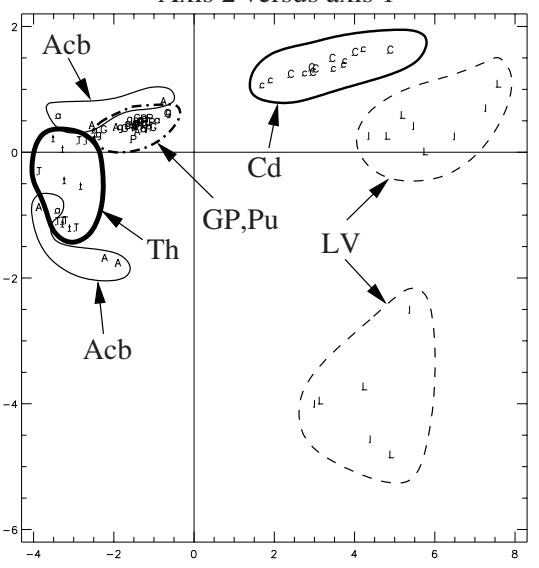

Axis 3 versus axis 1

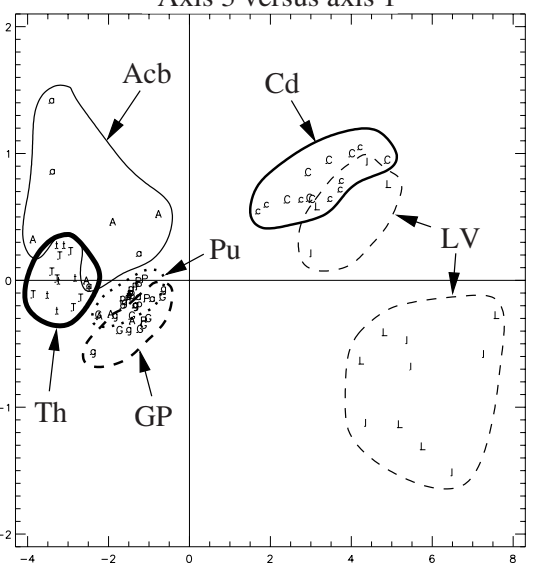

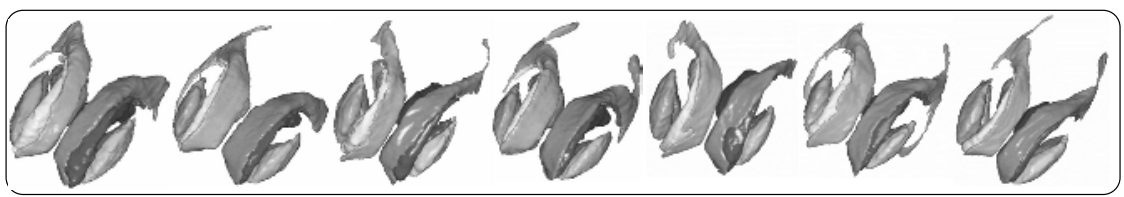

Fig. 2. The 84 deep anatomical objects used to analyze the shape representation provided by the $3 D$ invariants. The 12 invariants have been computed for each nucleus. Then a standard PCA has been performed and each nucleus is plotted in two charts corresponding to the 3 principal axes of the PCA. Abbreviations denote Acb: accumbens, Th: thalamus, Cd: caudate, GP: globus pallidus, $P$ u: putamen, LV: lateral ventricle. For each point, lower and upper case letters denote left and right hemispheres.

The size of each sulcus was computed from its skeletonized representation. Then, left $(\mathrm{L})$ and right $(\mathrm{R})$ sizes were used to obtain a normalized asymmetry index ((L$\mathrm{R}) /(\mathrm{L}+\mathrm{R}) / 2$ ) for each sulcus and each brain. For each sulcus, the Mann-Whitney U Test was used to compare the asymmetry indices of left-handed and right-handed groups. This test relies on rank order statistics, which are robust to potential outliers stemming from sulcus recognition errors. Several significant differences were revealed by our analysis ( $p<0.05$, not corrected for multiple comparisons), including most of the sulci of the motor areas: inferior precentral sulcus $(\mathrm{p}=0.013)$, intermediate precentral sulcus $(\mathrm{p}=0.019)$, central sulcus $(\mathrm{p}=0.031)$ [10].

To test if the $3 \mathrm{D}$ invariants could capture additional information about the handedness correlates on the folding patterns, the 12 invariants have been computed for each sulcus and each brain. For this computation, each sulcus is represented by a set of voxels of the global skeleton. For each sulcus, the Mann-Whitney U Test was used to compare the invariant distributions of left-handed and right-handed groups. It should be noted that this analysis involved $12 \times 116=1392$ tests, which calls for some correction for multiple testing. This correction however requires further work to take into account the complex dependences between these tests. With $p<0.001$, only two sulci yield significant results: the right inferior postcentral sulcus ( 2 invariants) and the left superior frontal sulcus ( 7 invariants). These sulci had not yielded significant size asymmetry index differences between both populations. Interestingly, with $(p<0.01)$, none of the sulci presenting 

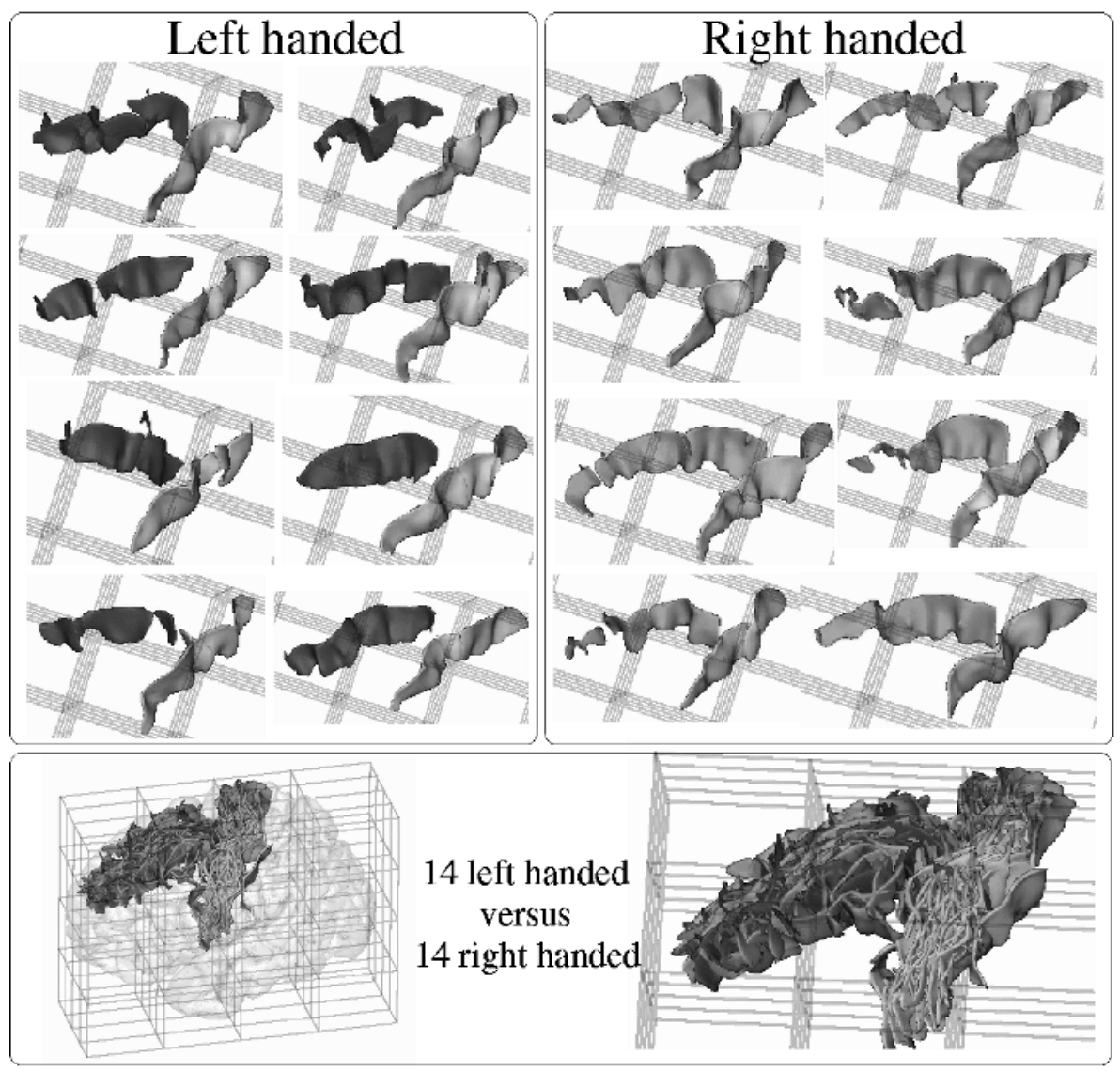

Fig. 3. top: The 8 superior frontal sulci (red or green) of both populations leading to the more extreme values for one of the $3 D$ invariants significantly correlated with handedness. The sulci of the right handed population are deeper near central sulcus (gold) than in the more frontal part. The grid corresponds to Talairach orientation. down: the same sulci for the 14 left handed subjects mixed with 14 right handed subjects matching for age and sex.

significant results with size asymmetry index led to results for 3D invariants, which tends to prove that these descriptors are really invariant for scale.

One of the difficulties with global shape descriptors like 3D invariants is the lack of simple interpretation in natural language terms. Considering the invariants as a first probe for exploratory analysis, however, such interpretation can be inferred by visual inspection of the extreme instances of both population. This has been done for the superior frontal sulcus using the invariant yielding the most significant result, leading to the fact that the sulcus of the right-handed population is deeper near the central sulcus than in the more frontal part (see Fig. 3). This observation may be related to models of the folding process, like the tension-based mechanism introduced by Van Essen [15].

Another experiment has consisted in gathering the sulci of the motor areas, which have led to handedness correlated asymmetry indices, to test if their global patterns 

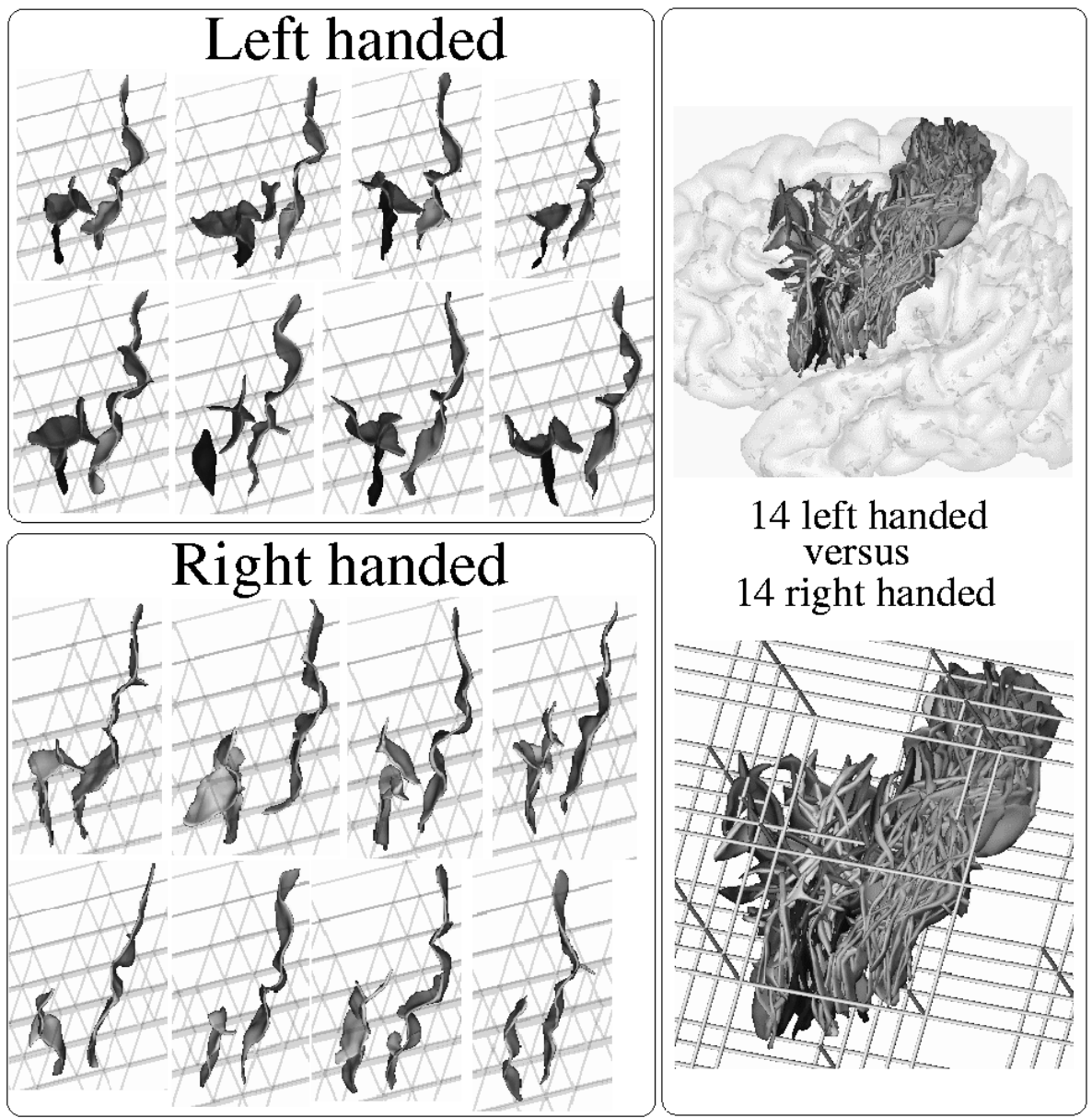

\section{4 left handed versus \\ 14 right handed}

Fig. 4. left: The 8 subjects of both populations leading to the more extreme values for one of the $3 D$ invariants, computed for the left motor complex, significantly correlated with handedness. The motor complex is made up of three sulci, which have yielded handedness correlated asymmetry indices [10]: central (cyan/gold), intermediate precentral (violet/yellow) and inferior precentral (blue/green) sulci. The intermediate precentral sulcus seems more parallel to the central sulcus in right handed subjects. right: the same sulci for the 14 left handed subjects mixed with 14 right handed subjects matching for age and sex. The central sulcus of left handed subjects seems shifted toward the back of the brain.

embed some handedness correlate. For this purpose, central, intermediate precentral and inferior precentral sulci have been merged into a "motor complex", for which the twelve invariants have been computed. These invariants have led to significant results for the left hemisphere $(\mathrm{p}=0.02)$. Visual inspection led to observe a specific pattern in the right handed population, namely an intermediate precentral sulcus more parallel to the central sulcus than in the left handed population (see Fig. 4). 


\section{Conclusion}

This paper has shown that invariants of 3D coordinate moments could allow the development of a sulcus based morphometry of the cerebral cortex. They provide shape descriptors that can be used to compare sulci with different topologies. Some more work, however, has to be done to deal with the correction for multiple testing. Anyway, this new morphometry strategy can already be used for exploratory purpose, in order to focus morphometric studies on specific regions of the folding patterns.

\section{References}

1. C. Brechbüler, G. Gerig, and O. Kübler. Parametrization of closed surfaces for 3D shape description. Computer Vision and Image Understanding, 61(2):154-170, 1995.

2. G. Burel and H. Hénocq. Three-dimensional invariants and their application to object recognition. Signal Processing, 45(1):1-22, July 1995.

3. D. L. Collins, P. Neelin, T. M. Peters et al. Automatic 3D intersubject registration of MR volumetric data in standardized talairach space. JCAT, 18(2):192-205, 1994.

4. A. R. Edmonds. Angular momentum in quantum mechanics. Princeton Univ. Press, 1960.

5. G.Gerig, M Styner, ME Shenton et al. Shape versus size: Improved understanding of the morphology of brain structures. In MICCAI 2001, LNCS 2208, Springer, pp 24-32, 2001.

6. M.-K. Hu. Visual pattern recognition by moment invariants. IRE Trans. Inf. Theory, 8:179187, February 1962.

7. G. Le Goualher et al. Statistical sulcal shape comparisons: application to the detection of genetic encoding of the central sulcus shape. Neuroimage, 11(5):564-574, 2000.

8. C.-H. Lo and H.-S. Don. 3D moment forms: their construction and application to object identification and positioning. IEEE PAMI, 11:1053-1064, October 1989.

9. J.-F. Mangin, V. Frouin, I. Bloch, et al. From 3D magnetic resonance images to structural representations of the cortex topography using topology preserving deformations. Journal of Mathematical Imaging and Vision, 5(4):297-318, 1995.

10. J.-F. Mangin, D. Rivière, A. Cachia et al. Object-based strategy for morphometry of the cerebral cortex. In IPMI, LNCS 2732, Springer Verlag, pp 160-171, 2003.

11. S. M. Pizer, D. S. Fritsch, P. A. Yushkevich et al. Segmentation, registration, and measurement of shape variation via image object shape. IEEE T. Med. Imaging, 18:851-865, 1999.

12. F. Poupon, J.-F. Mangin, V. Frouin, and I. Magnin. 3D multi-object deformable templates based on moment invariants. In 10th SCIA, volume I, pages 149-155, 1997.

13. F. Poupon et al. Multi-object Deformable Templates Dedicated to the Segmentation of Brain Deep Structures. In MICCAI'98, MIT, LNCS-1496, pp 1134-1143. Springer, 1998.

14. D. Rivière, J.-F. Mangin, et al. Automatic recognition of cortical sulci of the human brain using a congregation of neural networks. Med Image Anal, 6(2):77-92, 2002.

15. D. C. Van Essen. A tension-based theory of morphogenesis and compact wiring in the central nervous system. Nature, 385:313-318, 1997.

16. K. E. Watkins, T. Paus, J. P. Lerch, et al. Structural asymmetries in the human brain: a voxel-based statistical analysis of 142 mri scans. Cereb Cortex, 11(9):868-877, 2001. 